\title{
Analysis of an offset modulation transmission
}

\author{
Kahesh Dhuness ${ }^{*}$ and Bodhaswar T Maharaj
}

\begin{abstract}
In this article, a method called offset modulation (OM-OFDM) is proposed to control the peak-to-average power ratio (PAPR) of an orthogonal frequency division multiplexing (OFDM) signal. The theoretical bandwidth occupancy of the proposed offset modulated signal is derived. Using these bandwidth occupancy results, a closed-form theoretical bit error rate (BER) expression for an offset modulated transmission is derived and validated. Thereafter, a BER comparison between OM-OFDM and OFDM at a PAPR value of $13 \mathrm{~dB}$ shows that both methods offer similar BER characteristics for frequency selective fading channel conditions. The OM-OFDM method in addition is able to accurately control the PAPR of a transmission for a targeted BER. The authors have further proposed a newly applied power performance decision metric, which can be used throughout the PAPR field, in order to compare various methods. By using this power performance decision metric, the authors show that OM-OFDM offers between $4 \mathrm{~dB}-1.2 \mathrm{~dB}(60.34 \%-24.6 \%)$ and $4.1 \mathrm{~dB}-1.2 \mathrm{~dB}(60.8 \%-23.6 \%)$, net power performance gain (at a BER of $10^{-4}$ ) when compared to a clipped OFDM, OFDM, tone reserved (TR) OFDM and an active constellation extended (ACE) OFDM transmission in a frequency selective fading channel. Finally, by using a complementary cumulative distribution function (CCDF), the OM-OFDM method is shown to offer between $3.2 \mathrm{~dB}$ and $2 \mathrm{~dB}$ PAPR reduction (at a CCDF of $10^{-1}$ ) when compared to an OFDM, TR, clipped, and ACE OFDM transmission.
\end{abstract}

\section{Introduction}

Orthogonal frequency division multiplexing (OFDM) has become a very popular method for high-data rate communication, primarily due to its tight spectral efficiency and its robustness to multi-path fading. This has led to it being deployed in various standards, such as digital subscriber lines, digital video broadcasting (DVB), worldwide inter-operability for microwave access IEEE 802.16d standard and recently in long-term evolution. However, it is a well-known fact that OFDM is plagued by a large peakto-average power ratio (PAPR). This high PAPR occurs when the sinusoidal signals of the sub-carriers are added constructively. This results in an OFDM signal, which contains a number of infrequent peaks, which needs to be amplified before transmission through a channel. These high peaks necessitate the need for over-designed power amplifiers. Since these peaks are irregular, this leads to inefficient use of the power amplifiers, which ultimately leads to inefficient transmitters, as well as reduced battery life of the mobile device. Various methods [1-3] have been suggested to reduce the PAPR, such as

*Correspondence: kdhuness@ieee.org

Department of Electrical, Electronic and Computer Engineering, University of Pretoria, Pretoria, South Africa clipping, decision-aided reconstruction (DAR) clipping, coding, partial transmission sequence, selective mapping (SLM), nonlinear companding transforms, active constellation extension, tone reservation and constant envelope OFDM phase modulation, amongst others.

Clipping is the simplest method of reducing the PAPR, by limiting the peak amplitude level of the input signal to a predetermined level. At the receiver, the clipped samples can be reconstructed by using a number of methods $[1,2,4,5]$. Kim and Stuber [5] have recommended using an iterative process called DAR to reconstruct the clipped signal. A limiting factor of clipping, as well as DAR clipping, is that as the number of peak amplitudes increases, this would lead to a severe bit error rate (BER) degradation. Also, the iterative nature of the DAR technique requires increased computational complexity.

In contrast to clipping, coding can also be used to reduce the PAPR, by selecting a codeword which minimizes the PAPR. Various coding schemes have been recommended by Jiang and $\mathrm{Wu}$ [2] and Jones et al. [6]. Davis and Jedwab [7] have further shown that it is possible to combine block coding (with its encoding, decoding, and error-correcting capability) and Golay contemporary sequences (with their attractive PAPR properties), in order to reduce the PAPR. Coding can be used to reduce the

\section{Springer}

(c) 2013 Dhuness and Maharaj; licensee Springer. This is an Open Access article distributed under the terms of the Creative Commons Attribution License (http://creativecommons.org/licenses/by/2.0), which permits unrestricted use, distribution, and reproduction in any medium, provided the original work is properly cited. 
PAPR; however, it is not always possible to achieve a specific PAPR value. In certain cases coding gain is sacrificed for this PAPR decrease. An alternative method employed in PAPR reduction is the partial transmitted sequence (PTS) technique. In this PTS technique, the input data block is partitioned into disjointed sub-blocks. These sub-blocks are inverse fast Fourier transformed, thereafter these partial sequence sub-blocks are independently phase-rotated. The objective of this phase rotation is eventually to optimally combine these sub-blocks, to achieve a minimum PAPR. A limiting factor of PTS is that it requires high computational overhead to find an optimum phase-rotated sub-block combination and requires additional side information to be transmitted to allow the receiver to reconstruct the original signal.

In SLM, the input data block is mapped onto different candidate data blocks, all representing the same information as the original data block. These subsequent mapped data blocks are inverse fast Fourier transformed and the data transmission with the lowest PAPR is then selected for transmission [8]. Just as in the case of PTS, this method requires high computational overhead, as well as the transmission of side information. Wang and Ouyang [9] have proposed a method of reducing the computational complexity, while Breiling et al. [10] have suggested a method which does not require the transmission of side information. Despite all these methods, both PTS and SLM still require relatively high computational overhead and in some cases the transmission of side information.

Another method employed in PAPR reduction involves using a nonlinear companding transform. The idea behind nonlinear companding transforms originates from speech processing. Similar to speech signals, OFDM signals contain peaks which occur infrequently, thus similar companding techniques used in speech processing may be applied to improve the PAPR of an OFDM transmission. Wang et al. [11] have proposed using a nonlinear transform, which enlarges the small signals while compressing the large signals. Later Huang et al. [12] proposed a companding method based on $\mu$ law companding, which combined clipping and Wang companding, in order to reduce the PAPR of OFDM signals. Jiang and Zhu [13] have also proposed an alternative companding technique, which uses the statistical distribution of an OFDM transmitted signal to reduce the PAPR. These companding methods increase the average power of the signal and require larger linear amplifiers.

A further PAPR reduction method is active constellation extension (ACE) [14]. In ACE, the outer region of a constellation is intelligently extended outwards in order to reduce the PAPR. Extending the outer constellation points leads to an average power increase. Furthermore, extending the constellation intelligently requires the use of an iterative clipping process. The iterative nature of this process increases the computational complexity. Also the optimum choice of clipping parameters may prove difficult as well.

Another method used in PAPR reduction is tone reservation (TR) [15]. In TR, the transmitter does not send data on a specified set of sub-carriers. The values of these sub-carriers (which are determined by using an iterative clipping process) are chosen in order to reduce the PAPR of a transmission. Similar to the ACE method, the iterative nature of the TR process increases the computational complexity and the optimum choice of the clipping parameter may prove difficult. Furthermore, the reservation of sub-carriers compromises throughput.

In contrast to tone reservation, constant envelope OFDM phase modulation (CE-OFDM) [16-24] combines OFDM and phase modulation. The OFDM signal is phasemodulated, producing a constant envelope waveform. A draw-back of CE-OFDM is its bandwidth expansion. Narrow band constraining of the transmission affects the BER of the system (the classical feature of a phasemodulated signal). Ideally, a PAPR method which does not suffer from the draw-backs being experienced by current methods in the field is required. A method is required which meets the requirements summarized in Table 1.

In this article, the authors propose a method called offset modulation (OM-OFDM), which meets a number of the requirements summarized in Table 1 . The proposed offset modulation method is developed in Section 2. Thereafter, in Section 3, a closed-form bandwidth occupancy expression of an OM-OFDM transmission is derived. Using these bandwidth occupancy results, in Section 4, a closed-form BER expression for an OM-OFDM transmission is derived and validated. In Section 5, a newly applied power performance decision

\section{Table 1 An ideal PAPR reduction method}

\begin{tabular}{ll}
\hline Requirements & $\begin{array}{l}\text { Methods not complying } \\
\text { to the requirements }\end{array}$ \\
\hline Low complexity & PTS \\
& SLM, DAR clipping \\
& ACE, TR \\
\hline Does not lead to an increase in aver- & Nonlinear companding \\
age power & ACE \\
\hline Does not affect the coding gain & Coding \\
\hline $\begin{array}{l}\text { Does not require any further band- } \\
\text { width expansion or the transmis- }\end{array}$ & CE-OFDM \\
sion of side information & PTS \\
\hline $\begin{array}{l}\text { Does not lead to a severe BER } \\
\text { degradation as the }\end{array}$ & SLM, TR \\
number of carriers increases & Clipping \\
\hline
\end{tabular}


metric is presented which can be used throughout the PAPR field, in order to compare various methods. Thereafter, in Section 6, OFDM, OM-OFDM, clipped OFDM, ACE, and TR methods are compared by using a BER performance analysis, the newly applied power performance decision metric, and a complementary cumulative distribution function (CCDF). In Section 7, conclusions are drawn.

The contribution of this article is the introduction of a method called offset modulation which is used to control the PAPR of an OFDM transmission for a targeted BER. Both the theoretical bandwidth occupancy and BER expressions for an OM-OFDM transmission are derived. A further contribution is the introduction of a newly applied decision metric, which can be used throughout the PAPR field to compare various methods.

\section{Proposed offset modulation}

Consider the complex output of an $N$-point inverse Fourier transform OFDM signal, given by

$$
m(t)=\frac{1}{\sqrt{N}} \sum_{k=0}^{N-1} X_{k} e^{j \frac{2 \pi t k}{T_{s}}}, \quad 0 \leq t<T_{\mathrm{s}}
$$

where $T_{\mathrm{s}}$ is the symbol duration and $X_{k}$ represents the complex signal, which may also be written as $a_{k}+j b_{k}$, this signal may be modulated using the method which follows.

$$
\Phi_{1}(t)=\frac{\Re(m(t))}{\varsigma} \text { and } \Phi_{2}(t)=\frac{\Im(m(t))}{\varsigma}
$$

where $\Re$ and $\mathfrak{I}$, refer to the real and imaginary parts of the OFDM message signal, respectively, $\varsigma$ refers to a constant division term, whereas $\Phi_{1}(t)$ and $\Phi_{2}(t)$ represent the equivalent real and imaginary OFDM phase mappings, respectively. The real and imaginary components of the OFDM signal are divided by a constant $(\varsigma)$ term, this process ensures that the receiver can accurately extract these real and imaginary terms at the receiver. These $\Phi_{1}(t)$ and $\Phi_{2}(t)$ terms may now be combined into a unique co-sinusoid:

$$
\cos \left(2 \pi f_{c} t+\Phi_{1}(t)+\Psi_{o s}\right)-\cos \left(2 \pi f_{c} t+\Phi_{2}(t)\right)
$$

this expression may also be written as

$$
\begin{aligned}
& 2 \sin \left(\frac{\Phi_{2}(t)-\Phi_{1}(t)-\Psi_{\mathrm{os}}}{2}\right) \\
& \cdot \sin \left(2 \pi f_{c} t+\frac{\Phi_{1}(t)+\Psi_{\mathrm{os}}+\Phi_{2}(t)}{2}\right)
\end{aligned}
$$

where $\Psi_{\text {os }}$ refers to an offset term. The unique co-sinusoid combination in Equation (3), together with the choice of $\Psi_{\text {os }}$ and $\varsigma$ terms ensure that at the receiver the transmitted real and imaginary terms can be extracted. In this type of modulation, the parameters $\left(\Psi_{\mathrm{os}}, \varsigma\right)$ are chosen such that $\Psi_{\mathrm{os}}>\Phi_{2}(t)-\Phi_{1}(t)$, if $\Psi_{\mathrm{os}}$ is sufficiently large and $\Phi_{2}(t), \Phi_{1}(t)$ are sufficiently small. This implies that the $\Psi_{\mathrm{os}}$ term will dominate the expression, hence the name offset modulation (OM-OFDM) is proposed to describe this operation. A block diagram, depicted in Figure 1, shows the possible processes involved during an OM-OFDM transmission. The message signal is inverse fast Fourier transformed and up-sampled to produce an OFDM message signal. This OFDM message signal is then modulated using an OM modulator (Figure 2), thereafter the signal is passed through a channel. After which the incoming signal is passed through an OM demodulator (Figure 3) to recover the OFDM signal. The OFDM signal is then down sampled and fast Fourier transformed. Thereafter, equalization is performed to mitigate the channel effects.

Thus far, the proposed method may appear to be similar, if not identical, to phase modulation of an OFDM signal (CE-OFDM), which has been well documented [1624]. However, there are substantive differences between the two methods. For instance, the resultant CE-OFDM signal is spectrally noisy and is ideally suited for constellations without imaginary components (e.g., BPSK). In cases where imaginary components exist (e.g., as in 64-QAM), this constellation, as depicted in Figure 4, is uniquely mapped onto a different constellation without imaginary components (e.g., 64-QAM to 64-PAM mapping). Such a mapping process results in a severe BER degradation [25]. Hence, CE-OFDM has the ideally required $3 \mathrm{~dB}$ PAPR (which is permanently fixed), but the price paid for this is a severe BER degradation and a spectrally noisy signal. OM-OFDM handles constellations containing both real and imaginary components. The real and imaginary components are combined in a unique manner; the $\Psi_{\text {os }}$ and $\varsigma$ terms ensure that the receiver can successfully detect the original transmitted signal. The transmission may appear to be a phase-modulated signal; therefore, losing its attractive OFDM properties. However, the OM-OFDM system's transmitter-receiver structure (Figure 1) maintains the fundamental OFDM building blocks. The OMOFDM equalization process is identical to that employed in OFDM. Channel state information (CSI) is extracted from the pilot symbols and used during the equalization process to mitigate the effects of fading. Thus, the OMOFDM method still maintains the ease of equalization, whereas the CE-OFDM method requires a more complex equalization process.

During a CE-OFDM transmission, as depicted in Figure 4, a frequency-domain equalizer (FDE) is used to mitigate the effects of a channel. The FDE extracts CSI from the prefix [pilot and guard intervals (GI)], which are inserted between successive CE-OFDM blocks. During the FDE process either a zero-forcing or minimum mean-squared error equalizer can be used. The CE-OFDM equalization process requires additional overhead (pilot and GI) and an increase in computational 


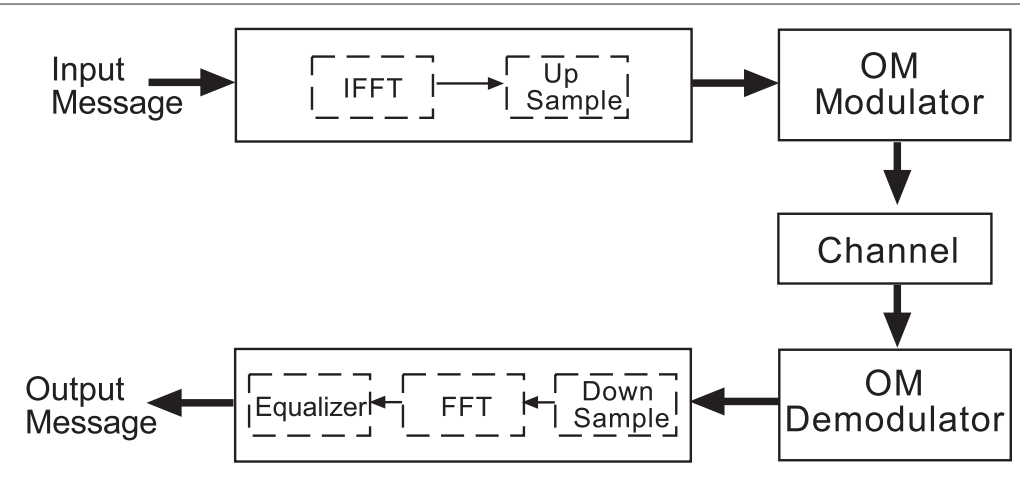

Figure 1 Transmitter-receiver structure.

complexity when compared to an OM-OFDM transmission. A comparison between Figures 1 and 4 demonstrates the structural difference between an OM-OFDM and CEOFDM transmission, in particular the placement of the equalizer. The only similarity that OM-OFDM and CEOFDM share is that both methods involve a form of phase modulation. Other than that, the two methods are significantly different. The OM-OFDM transmission in addition contains a dominant component. By subtracting from the dominant frequency component at the transmitter (Figure 2), and re-instating the subtracted term at the receiver (Figure 3), the PAPR may be controlled. This is not the case in an OFDM and CE-OFDM transmission. As the dominant component becomes prominent, the PAPR of the signal decreases. However, because in reality some energy restrictions are imposed on a transmitter, the other components can contain less energy, leading to a BER trade-off.

\section{Bandwidth occupancy of offset modulation}

In this Section, the bandwidth occupancy of an OMOFDM transmission may be investigated by considering a discrete complex OFDM signal, which is an extension of Equation (1) $\left(t=\frac{n T_{\mathrm{s}}}{N}\right)$, giving

$$
m_{n}=\frac{1}{\sqrt{N}} \sum_{k=0}^{N-1} X_{k} e^{j \frac{2 \pi n k}{N}}=\frac{1}{\sqrt{N}} \sum_{k=0}^{N-1} X_{k} e^{j w_{n} k} .
$$

In Equation (5), $w_{n}$ is an arbitrary chosen variable used to simplify the analysis, this equation may also be written as

$$
m_{n}=\frac{1}{\sqrt{N}} \sum_{k=0}^{N-1}\left(a_{k}+j b_{k}\right)\left(\cos \left(w_{n} k\right)+j \sin \left(w_{n} k\right)\right) .
$$

It can then be shown that

$$
\begin{aligned}
& \Phi_{1 n}=\frac{\Re\left[m_{n}\right]}{\varsigma} \approx \sum_{k=0}^{N-1} \beta_{1} \cos \left(w_{n} k\right)-\beta_{2} \sin \left(w_{n} k\right) \text { and } \\
& \Phi_{2 n}=\frac{\Im\left[m_{n}\right]}{\varsigma} \approx \sum_{k=0}^{N-1} \beta_{2} \cos \left(w_{n} k\right)+\beta_{1} \sin \left(w_{n} k\right),
\end{aligned}
$$

where $\Phi_{1 n}$ and $\Phi_{2 n}$ are the equivalent real and imaginary discrete OFDM phase mappings, respectively, $\beta_{1}$ and $\beta_{2}$ are constants defined as the adapted real and imaginary phase deviation of an OM-OFDM signal, respectively. After incorporating this into the unique co-sinusoidal (Equation 3), the following expression is obtained

$$
\begin{aligned}
u_{n}= & \Re\left[e^{j\left(2 \pi f_{c} n+\Psi_{o s}+\sum_{k=0}^{N-1} \beta_{1} \cos \left(w_{n} k\right)-\beta_{2} \sin \left(w_{n} k\right)\right)}\right] \\
& -\Re\left[e^{j\left(2 \pi f_{c} n+\sum_{k=0}^{N-1} \beta_{2} \cos \left(w_{n} k\right)+\beta_{1} \sin \left(w_{n} k\right)\right)}\right],
\end{aligned}
$$

Figure $2 \mathrm{OM}$ modulator structure. 


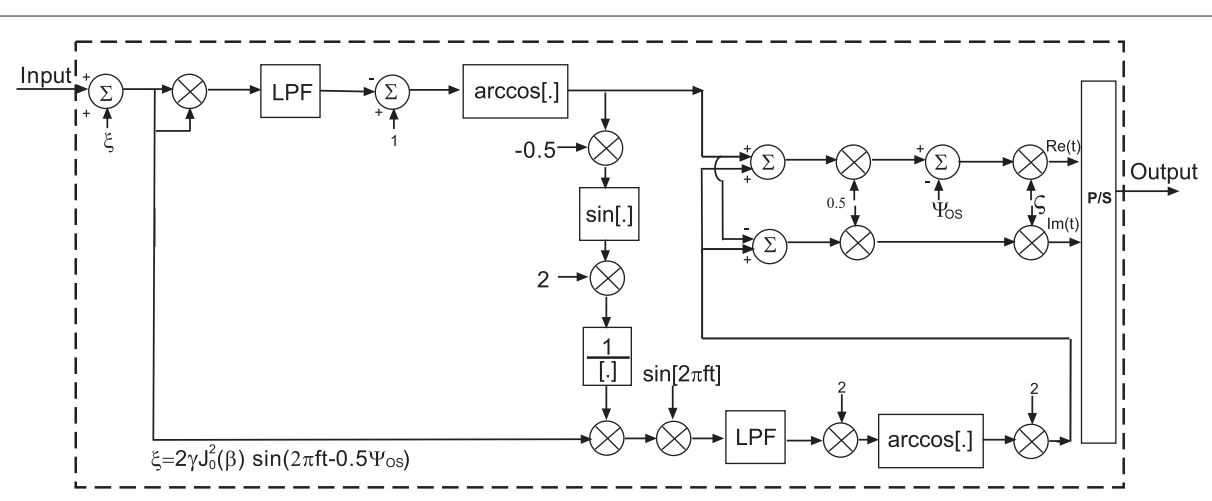

Figure $3 \mathrm{OM}$ demodulator structure.

in Equation (8), $u_{n}$ is the discrete signal which is to be transmitted. With the aid of Bessel functions [26], the Fourier series can be written as

$$
\begin{aligned}
e^{j\left(\beta \sin \left(w_{n} k\right)\right)} & =\sum_{l=-\infty}^{\infty} J_{l}(\beta) e^{j\left(l w_{n} k\right)} \text { and } \\
e^{j\left(\beta \cos \left(w_{n} k\right)\right)} & =\sum_{m=-\infty}^{\infty} J_{m}(\beta) e^{j\left(m w_{n} k+\frac{m \pi}{2}\right)} .
\end{aligned}
$$

Where $J_{l}(\beta)$ and $J_{m}(\beta)$ are Bessel functions of the first kind of order $l$ and $m$, respectively, with argument $\beta$. After substituting Equation (9) into Equation (8), it can then be shown that

$$
u_{n}=\prod_{k=0}^{N-1}\left(\left[\sum_{l=-\infty}^{\infty} \sum_{m=-\infty}^{\infty} J_{m}\left(\beta_{1}\right) J_{l}\left(\beta_{2}\right) \cos \right.\right.
$$

$$
\begin{aligned}
& \left.\times\left(\frac{2 \pi n k}{N}\left(f_{\mathrm{c}}+l+m\right)+\Psi_{\mathrm{os}}+\frac{m \pi}{2}\right)\right] \\
& -\left[\sum_{l=-\infty}^{\infty} \sum_{m=-\infty}^{\infty} J_{m}\left(\beta_{2}\right) J_{l}\left(\beta_{1}\right) \cdot \cos \right. \\
& \left.\left.\times\left(\frac{2 \pi n k}{N}\left(f_{\mathrm{c}}+l+m\right)+\frac{m \pi}{2}\right)\right]\right) .
\end{aligned}
$$

Here, $f_{\mathrm{c}}$ is the carrier frequency, when $l=m$ and $N=1$, Equation (10) may be simplified into

$$
\begin{gathered}
u_{n}=\sum_{y=0}^{2 x} \mid \sum_{z=0}^{2 x-y} 2 \sin \left(\frac{\pi(2 x-2 z-y) \pm 2 \Psi_{\mathrm{os}}}{4}\right) \\
\cdot J_{|-x+z|}\left(\beta_{1}\right)\left(\frac{\left|-x+z+\frac{1}{2}\right|}{-x+z+\frac{1}{2}}\right)^{|-x+z|}
\end{gathered}
$$

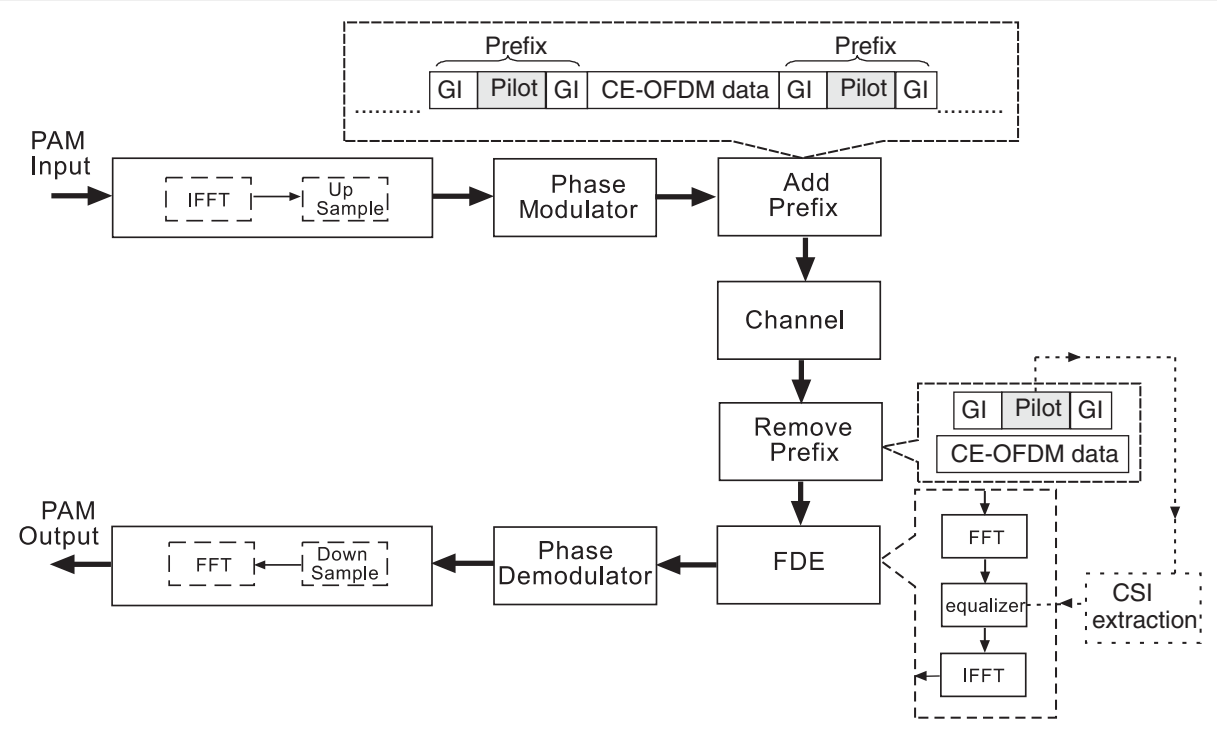

Figure 4 CE-OFDM transmitter-receiver structure. 


$$
\begin{aligned}
& \cdot J_{|x-y-z|}\left(\beta_{2}\right)\left(\frac{\left|x-y-z+\frac{1}{2}\right|}{x-y-z+\frac{1}{2}}\right)^{|x-y-z|} \\
& \cdot \sin \left(2 \pi\left(f_{\mathrm{c}}+y f_{d}\right)+\frac{2 \Psi_{\mathrm{os}} \pm y \pi}{4}\right) \mid
\end{aligned}
$$

where $f_{d}$ is an integer multiple of the modulation frequency and $2 x$ refers to an even number of frequency components of interest. In most cases the adapted phase deviation $\left(\beta_{1}\right.$ and $\left.\beta_{2}\right)$ of the signal is not known beforehand; however, a reasonably good approximation can be made, based on $[3,27,28]$

$$
\begin{aligned}
\alpha_{1} & =E[\max (|\Re(m(t))|)], \\
\alpha_{2} & =E[\max (|\Im(m(t))|)], \\
\beta_{1} & =\frac{\alpha_{1}}{\varsigma} \text { and } \\
\beta_{2} & =\frac{\alpha_{2}}{\varsigma} .
\end{aligned}
$$

In Equations (12) and (13), the $\alpha_{1}$ and $\alpha_{2}$ terms, respectively, refer to the real and imaginary phase deviations of the OFDM signal and $E[$.$] is the expected$ value. Typically, there is no interest in all the frequency components, but rather in the more dominant components. Hence, the bandwidth can be defined by considering only those sidebands which contain significant power. Suppose for explanation purposes the first two components $(2 x=2)$ are of interest and $\beta \approx$ $\beta_{1} \approx \beta_{2}$. Then by inspection of Equation (11), in Figure 5 for this particular case, the frequency spectrum and its corresponding amplitude components are shown.

This frequency spectrum in Figure 5 is different from that of a conventional phase-modulated signal. The squaring of the Bessel functions limits the bandwidth occupancy of the signal. If $\beta$ is sufficiently small $(\beta=$ $0.02)$, it can be seen that a large percentage of the power is constrained within these $(2 x=2)$ frequency components. This depiction in Figure 5 may serve as a simplistic OM-OFDM bandwidth occupancy description. The dominant frequency component is given by $2 J_{0}(\beta)^{2} \sin \left(2 \pi f_{c} t-\frac{\Psi_{o s}}{2}\right)$, provided $\Psi_{\mathrm{os}}>\Phi_{2}(t)-$ $\Phi_{1}(t)$. In such a case, the dominant frequency component can be shown to be dependent on the $\Psi_{\text {os }}$ term. This expression also provides some insight into an OMOFDM transmission, namely the bandwidth expansion is dependent on the $\varsigma$ term. The higher the $\varsigma$ term, the lower the phase $\beta$, thus indicating less bandwidth expansion. Ideally, an attempt might be made to choose $\varsigma$ as high as possible. However, as the $\varsigma$ term increases, the signal would lose resolution and this would lead to an increase in the BER. Thus far it has been shown that the dominant frequency component of an OM-OFDM transmission can be predicted by $2 J_{0}(\beta)^{2} \sin \left(2 \pi f_{c} t-\right.$ $\left.\frac{\Psi_{\mathrm{os}}}{2}\right)$. By subtracting $\gamma 2 J_{0}(\beta)^{2} \sin \left(2 \pi f_{\mathrm{c}} t-\frac{\Psi_{\mathrm{os}}}{2}\right), \quad 0 \leq$ $\gamma<1$ (where $\gamma$ is the dominant frequency component control factor) from the dominant frequency component at the transmitter (Figure 2) and re-instating the subtracted term at the receiver (Figure 3), the PAPR may be controlled. The receiver gains knowledge of the subtracted term by examining the PAPR of the incoming signal, from which the $\Psi_{\text {os }}, \varsigma$ and $\gamma$ terms can be extracted by using a simple look-up table. It might be argued that after a transmission through a multi-path fading channel, the received and transmitted PAPR might differ. However, for an $n$-tap channel, each path affects both the root mean square and peak value of the received signal equally, therefore the PAPR from each path is equivalent to the originally sent PAPR.

This principle can be demonstrated by using the $8 \mathrm{k}$ mode of the DVB-T2 standard [29], to transmit 64-QAM OM-OFDM data through a 5-tap typical-urban area [30] at a low $10 \mathrm{~dB}$ signal-to-noise ratio (SNR) $\left(E_{b} / N_{o}\right)$. The CCDFs of such a transmission, depicted in Figure 6,

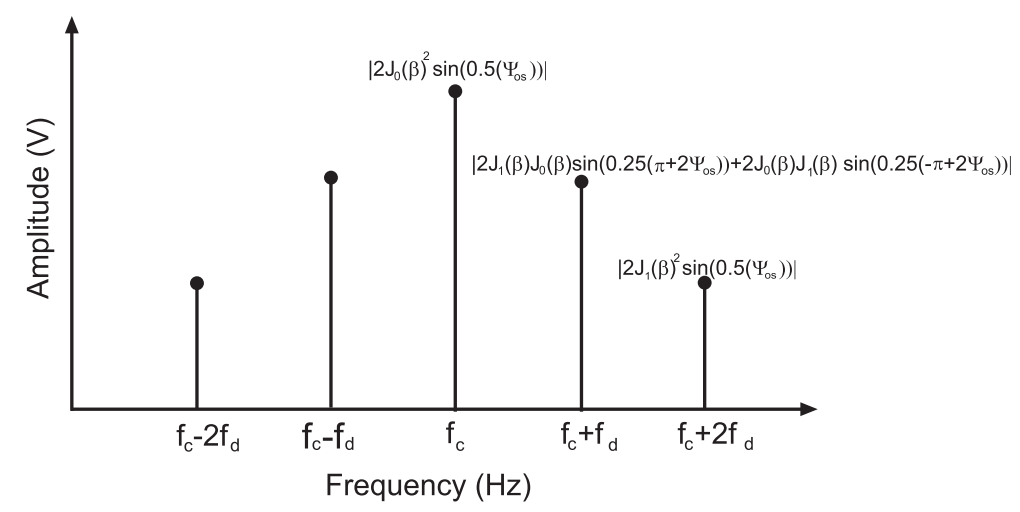

Figure 5 Theoretical derived (Equation 11) frequency spectrum of an OM-OFDM signal. 


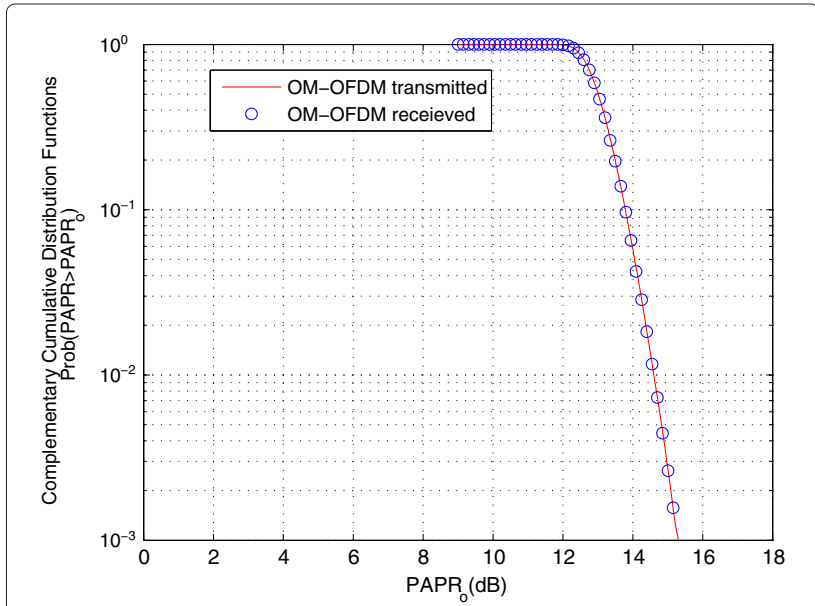

Figure 6 CCDFs for a 64-QAM constellation, when using the DVB-T2 standard for a 5-tap typical urban area at a $10 \mathrm{~dB}$ SNR $(\mathrm{Eb} / \mathrm{No})$.

indicates that the sent and received PAPR's are almost identical.

In the following section, the manner in which the dominant frequency component is varied and the resultant BER characteristics are presented.

\section{BER characteristics of offset modulation}

A received signal is expressed as $[21,31]$

$$
\begin{gathered}
r(t)=u(t)+n(t)= \\
u(t)+N_{c}(t) \cos \left(2 \pi f_{c} t\right) \\
-N_{s}(t) \sin \left(2 \pi f_{c} t\right),
\end{gathered}
$$

where $u(t)$ refers to an offset-modulated signal and $n(t)$ refers to the additive white Gaussian noise (AWGN). The $N_{c}(t)$ and $N_{s}(t)$ expressions refer to in-phase and quadrature components of noise, respectively. The noise expression can also be written as

$$
\begin{aligned}
n(t) & =\sqrt{N_{c}^{2}(t)+N_{s}^{2}(t)} \sin \left(2 \pi f_{\mathrm{c}} t+\arctan \frac{N_{c}(t)}{N_{s}(t)}\right) \\
& =V_{n}(t) \sin \left(2 \pi f_{c} t+\Phi_{n}(t)\right),
\end{aligned}
$$

where $V_{n}(t)$ and $\Phi_{n}(t)$ represent the envelope and phase of the band-pass noise. The offset modulated signal $u(t)$ can be expressed as indicated in Equation (18)

$$
\begin{aligned}
u(t)= & 2 \sin \left(\frac{\Phi_{2}(t)-\Phi_{1}(t)-\Psi_{\mathrm{os}}}{2}\right) \\
& \cdot \sin \left(2 \pi f_{\mathrm{c}} t+\frac{\Phi_{1}(t)+\Psi_{\mathrm{os}}+\Phi_{2}(t)}{2}\right) \\
= & A_{c} \sin \left(2 \pi f_{\mathrm{c}} t+\Phi(t)\right),
\end{aligned}
$$

where $A_{c}$ and $\Phi(t)$ can be seen as the envelope and phase of an offset modulated signal. If the assumption is made that the signal is much larger than the noise and after using phasor manipulation, it can be shown that the received signal can be written as

$$
\begin{aligned}
r(t) \approx & \left(A_{c}(t)+V_{n}(t) \sin \left(\Phi_{n}(t)-\Phi(t)\right)\right) \\
& \cdot \sin \left(2 \pi f_{\mathrm{c}} t+\Phi(t)+\frac{V_{n}(t) \cos \left(\Phi_{n}(t)-\Phi(t)\right)}{A_{c}}\right)
\end{aligned}
$$

From the equation above, it can be seen that the noise expression at one branch of the demodulator can be written as

$$
Y_{n}(t)=\frac{V_{n}(t) \cos \left(\Phi_{n}(t)-\Phi(t)\right)}{A_{c}} .
$$

Then by using the following identity,

$$
\cos (\alpha-\beta)=\cos \alpha \cos \beta+\sin \alpha \sin \beta
$$

the noise expression can be represented by

$Y_{n}(t)=\frac{V_{n}(t) \cos \left(\Phi_{n}(t)\right) \cos (\Phi(t))+V_{n}(t) \sin \left(\Phi_{n}(t)\right) \sin (\Phi(t))}{A_{c}}$

therefore

$$
Y_{n}(t)=\frac{n_{c}(t) \cos (\Phi(t))+n_{s} \sin (\Phi(t))}{A_{c}}
$$

where $n_{c}$ and $n_{s}$ refer to the noise co-sinusoidal and sinusoidal expressions, respectively. An auto correlation is performed on the noise, in order to study its spectrum characteristics. This can be expressed as follows

$$
\begin{aligned}
E\left[Y_{n}(t) Y_{n}(t+\tau)\right]= & E\left[\frac{n_{c}(t) n_{c}(t+\tau) \cos (\Phi(t)) \cos (\Phi(t+\tau))+n_{c}(t) n_{s}(t+\tau) \cos (\Phi(t)) \sin (\Phi(t+\tau))}{A_{c}{ }^{2}}\right. \\
& \left.\frac{+n_{s}(t) n_{c}(t+\tau) \sin (\Phi(t)) \cos (\Phi(t+\tau))+n_{s}(t) n_{s}(t+\tau) \sin (\Phi(t)) \sin (\Phi(t+\tau))}{A_{c}{ }^{2}}\right]
\end{aligned}
$$


According to [31], for stationary white noise,

$$
\begin{aligned}
n_{c}(t) n_{s}(t+\tau) & =n_{c}(t) n_{s}(t+\tau)=0 \\
R_{n c}(\tau) & =n_{c}(t) n_{c}(t+\tau) \\
R_{n s}(\tau) & =n_{s}(t) n_{s}(t+\tau) \\
R_{n s}(\tau) & =R_{n c}(\tau) .
\end{aligned}
$$

In Equations (25) and (26), respectively, $R_{n c}(\tau)$ and $R_{n s}(\tau)$ are bandpass signals. After substituting Equations (25), (26), (27), and (28) into Equation (24), this results in

$$
\begin{aligned}
E\left[Y_{n}(t) Y_{n}(t+\tau)\right]= & E\left[\frac{R_{n c}(\tau) \cos (\Phi(t)) \cos (\Phi(t+\tau))}{A_{c}^{2}}\right. \\
& \left.+\frac{R_{n s}(\tau) \sin (\Phi(t)) \sin (\Phi(t+\tau))}{A_{c}{ }^{2}}\right] .
\end{aligned}
$$

After using the identity presented in Equation (21) in conjunction with Equation (28), then Equation (29) simplifies to

$$
E\left[Y_{n}(t) Y_{n}(t+\tau)\right]=\frac{R_{n c}(\tau)}{A_{c}{ }^{2}} E[\cos (\Phi(t+\tau)-\Phi(t))] .
$$

According to [31], at any fixed time $t$, a random variable $Z(t, \tau)=\Phi(t+\tau)-\Phi(t)$ is the difference between two jointly Gaussian random variables, which is itself a Gaussian random variable with mean equal to zero and a variance given by

$$
\begin{aligned}
\sigma_{Z}^{2} & =E\left[(\Phi(t+\tau)-\Phi(t))^{2}\right] \\
& =E\left[\Phi^{2}(t+\tau)+\Phi^{2}(t)\right]-2 R_{\Phi}(\tau) \\
& =2\left(R_{\Phi}(0)-R_{\Phi}(\tau)\right) .
\end{aligned}
$$

As previously mentioned $2 R_{\Phi}(\tau)$ is a bandpass signal. Using Equation (30) in conjunction with Equation (31) results in

$$
\begin{aligned}
E\left[Y_{n}(t) Y_{n}(t+\tau)\right] & =\frac{R_{n c}(\tau)}{A_{c}{ }^{2}} E[\cos (\Phi(t+\tau)-\Phi(t))] \\
& =\frac{R_{n c}(\tau)}{A_{c}{ }^{2}} \Re\left(E\left[e^{-j(\Phi(t+\tau)-\Phi(t))}\right]\right) \\
& =\frac{R_{n c}(\tau)}{A_{c}{ }^{2}} \Re\left(E\left[e^{-j(Z(t, \tau))}\right]\right) \\
& =\frac{R_{n c}(\tau)}{A_{c}{ }^{2}} \Re\left(E\left[e^{-j\left(\frac{\sigma_{Z}}{2}\right)}\right]\right) \\
& =\frac{R_{n c}(\tau)}{A_{c}^{2}} \Re\left(E\left[e^{-\left(R_{\Phi}(0)-R_{\Phi}(\tau)\right)}\right]\right) \\
& =\frac{R_{n c}(\tau)}{A_{c}{ }^{2}}\left(E\left[e^{-\left(R_{\Phi}(0)-R_{\Phi}(\tau)\right)}\right]\right) .
\end{aligned}
$$

Then from the autocorrelation of the noise, the power spectral density of the noise can be written as

$$
\begin{aligned}
S_{Y}(f) & =\mathscr{F}\left[R_{Y}(\tau)\right]=\mathscr{F}\left[\frac{R_{n c}(\tau)}{A_{c}^{2}}\left(E\left[e^{-\left(R_{\Phi}(0)-R_{\Phi}(\tau)\right)}\right]\right)\right] \\
& =\frac{e^{-R_{\Phi}(0)}}{A_{c}^{2}} \mathscr{F}\left[R_{n c}(\tau) e^{R_{\Phi}(\tau)}\right] \\
& =\frac{e^{-R_{\Phi}(0)}}{A_{c}^{2}} \mathscr{F}\left[R_{n c}(\tau) g(\tau)\right]=\frac{e^{-R_{\Phi}(0)}}{A_{c}^{2}} S_{n c}(f) \star G(f) .
\end{aligned}
$$

In Equation (33), $G(f)$ is the Fourier transform $(\mathscr{F})$ of $g(\tau)=e^{R_{\Phi}(\tau)}, S_{n c}(f)$ is the Fourier transform of $R_{n c}$ and the $\star$ refers to a convolutional process. For a specific bandwidth $-\frac{B_{c}}{2}$ and $\frac{B_{c}}{2}$, for this case, $S_{n c}(f)=N_{o}$, here $N_{o}$ is the power spectral density of the additive noise

$$
\begin{aligned}
S_{Y}(f) & =\frac{e^{-R_{\Phi}(0)}}{A_{c}^{2}} N_{o} \int_{\frac{B_{c}}{2}}^{\frac{-B_{c}}{2}} G(f) d f \approx \frac{e^{-R_{\Phi}(0)}}{A_{c}^{2}} N_{o} \int_{\infty}^{-\infty} G(f) d f \\
& =\left.\frac{e^{-R_{\Phi}(0)}}{A_{c}^{2}} N_{o} g(\tau)\right|_{\tau=0}=\frac{e^{-R_{\Phi}(0)}}{A_{c}^{2}} N_{o} e^{R_{\Phi}(0)}=\frac{N_{o}}{A_{c}^{2}} .
\end{aligned}
$$

Suppose $A_{c}(t) \approx 2 \sin \left(\frac{-\varphi}{2}\right)$ (Equation 18), where $\varphi$ refers to a constant term. After using Equation (34) and by following a similar methodology, the $Y$ and $X$ output power spectral density of the noise component can be written as follows

$$
S_{Y}(f) \approx \frac{N_{o}}{4 \sin ^{2}\left(\frac{-\varphi}{2}\right)} \quad \text { and } \quad S_{X}(f) \approx N_{o}
$$

After band $\left(B_{c}\right)$ limiting the transmission $\left(0>B>\frac{B_{c}}{2}\right)$ the noise variance at the output of the various branches of the demodulator (Figure 1) is given by

$$
\sigma_{Y}^{2} \approx \frac{N_{o}}{8 \sin ^{2}\left(\frac{-\varphi}{2}\right)} \quad \text { and } \quad \sigma_{X}^{2} \approx \frac{N_{o}}{2}
$$

Here $\varphi$ may be approximated by Table 2 . In Table $2, \gamma$ is the dominant frequency component control factor and the $\alpha\left(\alpha \approx \alpha_{1} \approx \alpha_{2}\right)$ and $\beta\left(\beta \approx \beta_{1} \approx \beta_{2}\right)$ terms originate from Equations (12), (13), (14), and (15). The proposed $\varphi$ term is obtained, based on a number of observations, provided $0 \leq \varphi \leq \frac{\pi}{2}$, namely

$$
\begin{aligned}
& N_{o} \propto \frac{1}{\sin \left(\Psi_{\mathrm{os}}\right)} \\
& N_{o} \propto \varsigma \\
& N_{o} \propto[1-\gamma] .
\end{aligned}
$$

\section{Table 2 Selection of a $\varphi$ term, based on $\gamma$ and $\alpha$}

\begin{tabular}{llll}
\hline & $0<\boldsymbol{\alpha}<0.1$ & $\mathbf{0 . 1} \leq \boldsymbol{\alpha}<\mathbf{0 . 2}$ & $\mathbf{0 . 2} \leq \boldsymbol{\alpha}<\mathbf{0 . 3}$ \\
\hline$\varphi \approx \frac{\beta \sin \left(\Psi_{\mathrm{os}}\right)}{4(1-\gamma)}$ & $0 \leq \gamma<0.988$ & $0 \leq \gamma<0.97$ & $0 \leq \gamma<0.96$ \\
$\varphi \approx \frac{\beta \sin \left(\Psi_{\mathrm{os}}\right)}{5(1-\gamma)}$ & $0.988 \leq \gamma<1$ & $0.97 \leq \gamma<1$ & $0.96 \leq \gamma<1$ \\
\hline
\end{tabular}


From these observations, the $\varphi$ term in Table 2 is proposed. Therefore, from the $\gamma$ and $\alpha$ terms of a particular transmission, either the $\varphi \approx \frac{\beta \sin \left(\Psi_{\mathrm{os}}\right)}{4(1-\gamma)}$ or $\varphi \approx \frac{\beta \sin \left(\Psi_{\mathrm{os}}\right)}{5(1-\gamma)}$ term can be used to describe the noise properties. Based on Equation (36), by using [32] an approximate theoretical BER expression for a QAM and PSK OM-OFDM transmission through an AWGN channel can be written as shown in Equations (40), (41), and (42).

$$
\begin{aligned}
\mathrm{BER}_{\mathrm{EVEN}} \approx & \frac{k \cdot \ell(M-2)+\wp \cdot k(M-2 \sqrt{M}+2)}{M \cdot k^{2}} \\
& +\frac{\wp \cdot \ell(2 \sqrt{M}-M-1)}{M \cdot k^{2}}, \\
\mathrm{BER}_{\mathrm{ODD}} \approx & \frac{\left(M-2-\left[\frac{3 \sqrt{2} M-8 \sqrt{M}}{2 \sqrt{M}}\right]\right) \wp \cdot k}{M \cdot k^{2}} \\
& +\frac{(M-2) k \cdot \ell-\left(M-3-\left[\frac{3 \sqrt{2} M-8 \sqrt{M}}{2 \sqrt{M}}\right]\right) \wp \cdot \ell}{M \cdot k^{2}},
\end{aligned}
$$

$$
\operatorname{BER}_{\mathrm{PSK}} \approx \operatorname{erfc}\left(\sqrt{\frac{4 k \cdot E_{b} \sin ^{2}\left(\frac{-\varphi}{2}\right) \sin ^{2}\left(\frac{\pi}{M}\right)}{N_{o}\left(\sin ^{2}\left(\frac{-\varphi}{2}\right)+1\right)}}\right),
$$

where

$$
\begin{aligned}
& \wp=\operatorname{erfc}\left(\sqrt{\frac{k E_{b}}{\xi_{a v} N_{o}}}\right) \text { and } \\
& \ell=\operatorname{erfc}\left(\sqrt{\frac{4 k \sin ^{2}\left(-\frac{\varphi}{2}\right) E_{b}}{\xi_{a v} N_{o}}}\right) .
\end{aligned}
$$

Here, $E_{b}$ refers to the energy per bit, $\frac{E_{b}}{N_{o}}$ refers to the SNR per bit, $M$ denotes $M$-ary levels and $M=2^{k}$. Also, $\operatorname{erfc}$ denotes the error function and $\xi_{a v}$ is defined as the energy per symbol. The $\wp$ and $\ell$ terms are arbitrarily chosen variables used to simplify the BER expression. The expressions in Equations (40) and (41) apply to a rectangular QAM constellation when $k$ is even and $k$ is odd, respectively. Similarly, the expression in Equation (42) applies to a PSK constellation. It should be noted that the theoretical expressions do not accept a $\gamma=1$; if such a term arises, then $\varphi=1$ (BER EVEN $), \varphi=1.5$ (BER $\mathrm{ODD})$, and $\varphi=1.23$

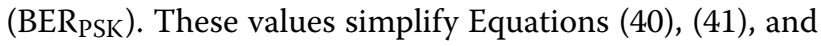
(42) into a BER expression for an AWGN transmission. Various QAM constellation BER expressions, summarized in Table 3, were obtained when using Equations (40) and (41).

For the choice of $\Psi_{\mathrm{os}}, \varsigma$, and $\gamma$ terms, both Equation (36) and Table 2 (provided $0 \leq \varphi \leq \frac{\pi}{2}$ ) offer guidelines for these parameters. An attempt might be made to reduce the noise component (Equation 36) by increasing the $\Psi_{\text {os }}$ term. Ideally, a $\Psi_{\text {os }}$ value as close as possible to the limits $\left(\Psi_{\mathrm{os}}=-\frac{\pi}{2}, \frac{\pi}{2}\right)$ should be used.
Table 3 Various QAM constellation BER expressions

\begin{tabular}{llll}
\hline QAM & $\boldsymbol{k}$ & $\boldsymbol{\xi}_{a v}$ & BER expressions \\
\hline 4 & 2 & 2 & $\frac{2 k \cdot \ell+2 \wp \cdot k-\wp \cdot \ell}{4 k^{2}}$ \\
8 & 3 & 14 & $\frac{6 k \cdot \ell+3 \wp \cdot \ell}{8 k^{2}}$ \\
16 & 4 & 10 & $\frac{14 k \cdot \ell+10 \wp \cdot k-9 \wp \cdot \ell}{10 k^{2}}$ \\
64 & 6 & 42 & $\frac{62 k \cdot \ell+50-49 \wp \cdot \ell}{64 k^{2}}$ \\
\hline
\end{tabular}

As $\Psi_{\text {os }}$ approaches the limits, as shown in Figure 7, this lowers the noise components. As the $\Psi_{\text {os }}$ term moves away from these limits, this leads to an increase in the BER degradation. It is also noted that as the $\varsigma$ term increases, the signal would lose resolution, which will affect the BER characteristics. Similarly, as the $\gamma$ term approaches 1, as shown in Figure 8, this leads to less noise, since the PAPR is being sacrificed to obtain this BER performance improvement.

Using these guidelines the theoretically derived expressions need to be validated. By means of a simulation (the $2 \mathrm{k}$ and the $8 \mathrm{k}$ modes of the DVB-T2 standard [29]), offset modulated 64-QAM (8k mode), as well as 8-PSK ( $2 \mathrm{k}$ mode) Gray-coded signal constellations were used to transmit data through an AWGN channel. The parameters used for the 8-PSK and 64-QAM OM-OFDM transmission are given in Tables 4 and 5 , respectively. In Tables 4 and 5 , the $\varphi$ term is calculated by substituting $\alpha$, $\varsigma, \Psi_{\mathrm{os}}$, and $\gamma$ terms into the $\varphi$ expression in Table 2. The specific terms ( $\zeta$ and $\Psi_{\text {os }}$ ) are chosen (as previously discussed in this section) to minimize the BER degradation, and the $\alpha$ term is obtained as indicated in Equations (12) and (13). In Tables 4 and 5, the various parameters for a $7 \mathrm{~dB}-13 \mathrm{~dB}$ PAPR range is presented. The $\gamma$ term can be further varied, until an average PAPR in the $3 \mathrm{~dB}-13 \mathrm{~dB}$ is

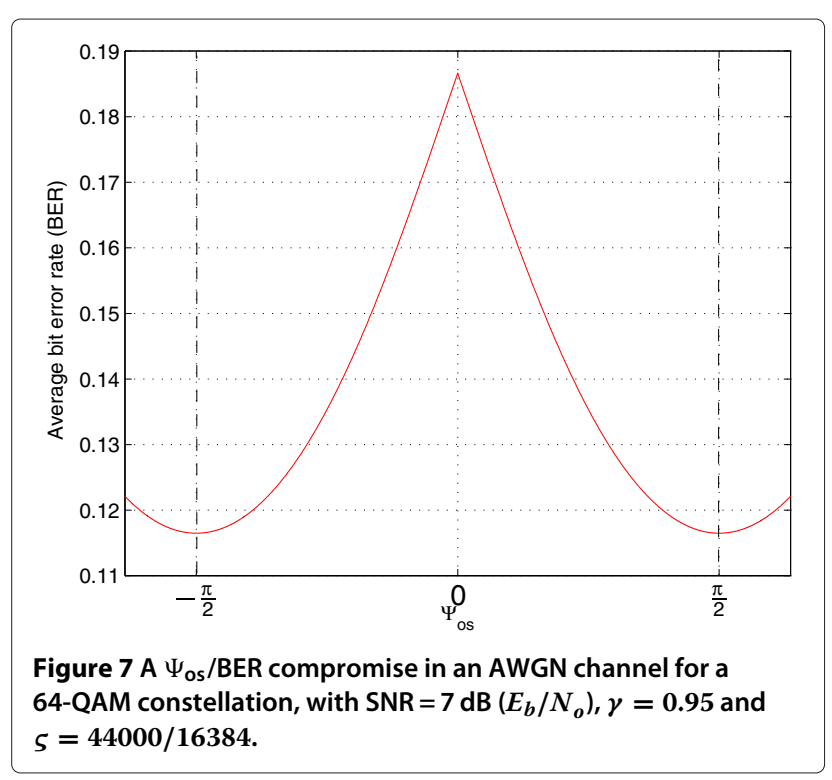




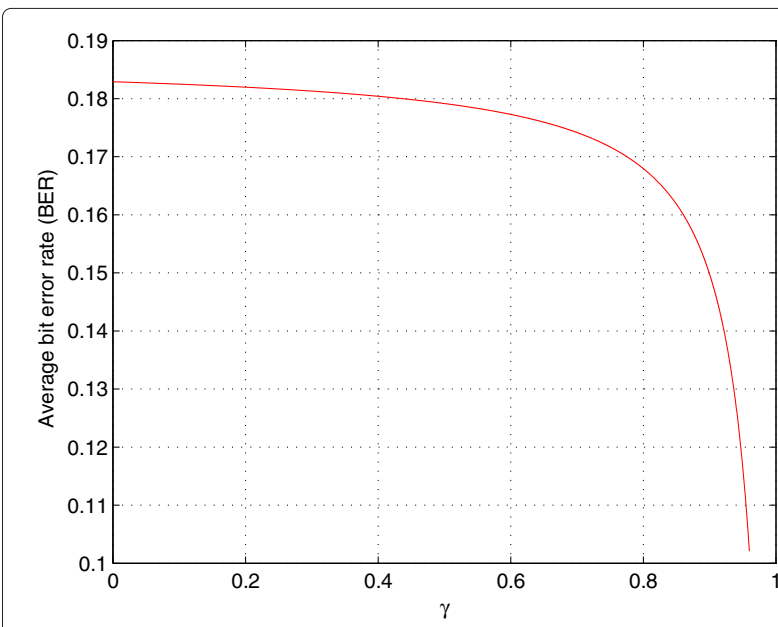

Figure $8 \mathrm{~A} \gamma / \mathrm{BER}$ compromise in an AWGN channel for a 64-QAM constellation, with $\mathrm{SNR}=7 \mathrm{~dB}\left(E_{b} / N_{o}\right), \Psi_{\mathrm{os}}=1.59$ and $\varsigma=44000 / 16384$.

reached. The lower bound ( $3 \mathrm{~dB})$ is the ideal average PAPR [21] and the upper bound (12 dB or $13 \mathrm{~dB})$ indicates the average PAPR of a traditional OFDM transmission [33] (since an attempt is made to reduce this PAPR). A BER comparison between the theoretically derived and simulated 8-PSK and 64-QAM OM-OFDM transmission is shown in Figures 9 and 10, respectively.

From this comparison, it is seen that the theoretically predicted results (Equations 40 and 42 ) and the simulated results correlate reasonably well, thus validating the theoretically derived expression. Furthermore, Figure 11 depicts the complementary CCDFs for a 64-QAM OMOFDM transmission. This CCDF graph can be interpreted as the probability of the various transmissions (in Figure 10) having a PAPR value above a certain threshold $\left(\mathrm{PAPR}_{\mathrm{o}}\right)$.

In the next section, a power performance decision metric is presented, which will be later used to highlight the benefits of using an OM-OFDM transmission.

\section{Decision metric}

Liang et al. [34] have proposed a decision metric $(D)$, to deal with the relationship between amplifier

Table 4 Parameters for an 8-PSK OM-OFDM system $(\alpha=0.027)$

\begin{tabular}{lllll}
\hline PAPR $(\mathbf{d B})$ & $\boldsymbol{\Psi}_{\text {os }}$ & $\boldsymbol{S}$ & $\boldsymbol{\gamma}$ & $\boldsymbol{\varphi}$ \\
\hline 7 & 1.5 & $10000 / 4096$ & 0.9889 & 0.2485 \\
8 & 1.5 & $10000 / 4096$ & 0.992 & 0.3 \\
9 & 1.5 & $10000 / 4096$ & 0.9941 & 0.4674 \\
10 & 1.5 & $10000 / 4096$ & 0.996 & 0.55 \\
11 & 1.5 & $10000 / 4096$ & 0.997 & 0.7 \\
12 & 1.5 & $10000 / 4096$ & 1 & 1.23 \\
\hline
\end{tabular}

Table 5 Parameters for an 64-QAM OM-OFDM system $(\alpha=0.27)$

\begin{tabular}{lllll}
\hline PAPR (dB) & $\boldsymbol{\Psi}_{\text {os }}$ & $\boldsymbol{S}$ & $\boldsymbol{\gamma}$ & $\boldsymbol{\varphi}$ \\
\hline 7 & 1.596 & $44000 / 16384$ & 0.86 & 0.2 \\
8 & 1.596 & $44000 / 16384$ & 0.9 & 0.251 \\
9 & 1.596 & $44000 / 16384$ & 0.925 & 0.34 \\
10 & 1.596 & $44000 / 16384$ & 0.943 & 0.44 \\
11 & 1.596 & $44000 / 16384$ & 0.962 & 0.53 \\
12 & 1.596 & $44000 / 16384$ & 0.97 & 0.67 \\
13 & 1.596 & $44000 / 16384$ & 1 & 1 \\
\hline
\end{tabular}

efficiency, amplifier distortion, signal bandwidth occupation, throughput, and power consumption. This metric can be adapted to investigate whether the proposed OM-OFDM transmission has an optimum solution and whether a net gain exists for such a solution. The decision metric is given by

$$
D=\frac{E_{t}}{N_{o}} \cdot \frac{W}{R_{b}},
$$

where $R_{b}$ is the data rate and $W$ refers to the bandwidth occupancy. Typically during fair comparisons, identical throughput and bandwidth occupancies are used, thus $\frac{W}{R_{b}}$ remains constant and the metric simplifies to

$$
D \propto \frac{E_{t}}{N_{o}} .
$$

where $E_{t}$ is the total energy per bit, for a specific BER. Unlike traditional approaches, which only take into account the received energy per bit $\left(E_{b}\right)$ and often ignore the total energy consumption (e.g., dc power consumption

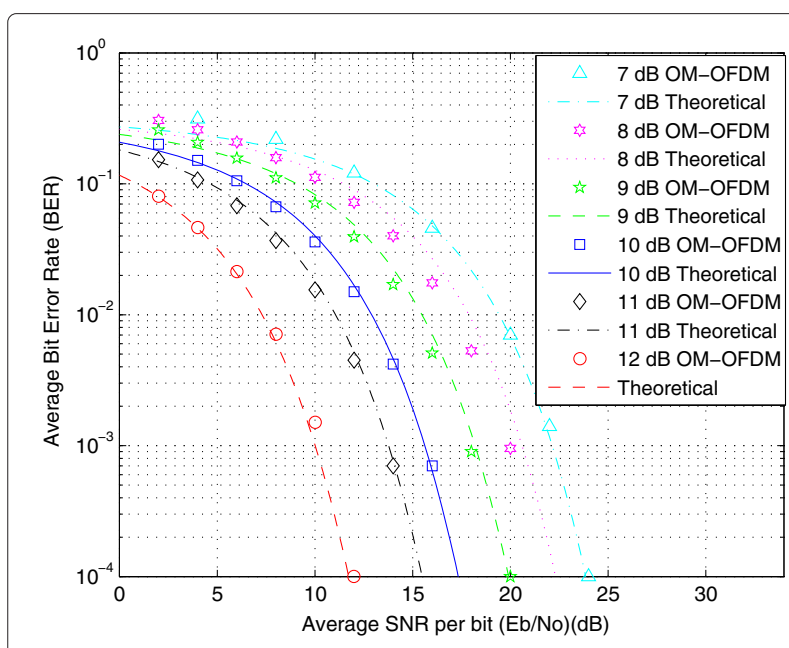

Figure 9 Theoretically derived (Equation 42) and simulated BER comparisons for an OM-OFDM transmission in an AWGN channel for an 8-PSK constellation. 


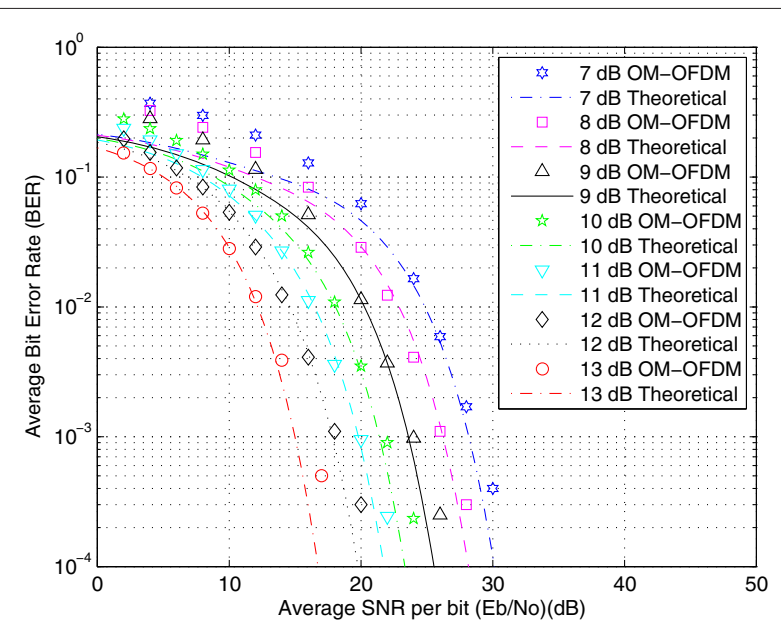

Figure 10 Theoretically derived (Equation 40) and simulated BER comparisons for an OM-OFDM transmission in an AWGN channel for a 64-QAM constellation.

of the amplifier), $E_{t}$ incorporates the total energy per bit and can be written as

$$
E_{t}=E_{b}+E_{w}
$$

In Equation (46), $E_{w}$ is the wasted energy per bit due to inefficient power amplifier utilization. In order to determine $E_{t}$, the power added efficiency (PAE) of the amplifier which is to be used is required. In the next section, this metric is applied and the benefits of using an OM-OFDM transmission are presented.

\section{Results and discussion}

A 64-QAM Gray-coded 8k mode of the DVB-T2 [29] standard was used to compare OFDM, ACE OFDM,

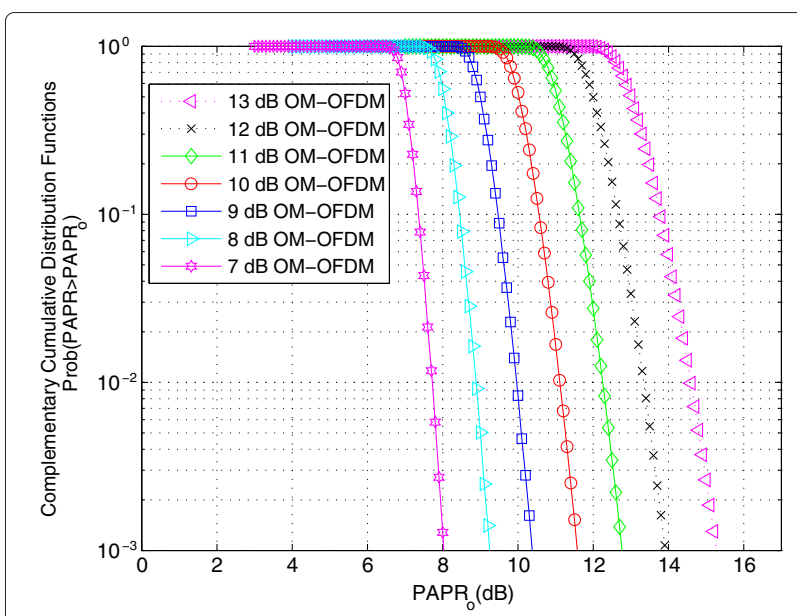

Figure 11 Complementary cumulative distribution functions for a 64-QAM constellation, when using the DVB-T2 standard. tone reserved (TR) OFDM, OM-OFDM, and a classically clipped OFDM transmissions. The clipping method was chosen since, to the best of the authors knowledge this method in conjunction with the OM-OFDM method are the only methods currently in the PAPR field, which allows for the accurate control of the PAPR of an OFDM transmission. The ACE and TR methods were selected since the DVB-T2 standard has recommended that these methods be used to reduce the PAPR of an OFDM transmission.

When classically clipping a signal at various clipping rates (CRs), both in-band and out-of-band distortions are introduced. In order to minimize the in-band distortion, the classically clipped OFDM signal was over-sampled by a factor of 4 . To limit the out-of-band distortion, the clipped OFDM signal was filtered before transmission with a $7^{\text {th }}$-order Butterworth band-pass filter, with a $9 \mathrm{~dB}$ ripple in the pass-band and a $42 \mathrm{~dB}$ stop attenuation.

The ACE method made use of the projection onto convex sets (POCS) $[35,36]$ approach. This iterative filtering and clipping ACE process involved using an oversampled signal (oversampled by a factor of 4 ), which is clipped with a clipping threshold of $7.8 \mathrm{~dB}$ and thereafter filtered with a 14th-order Butterworth band-pass filter, with a $9 \mathrm{~dB}$ ripple in the pass-band and a $42 \mathrm{~dB}$ stop attenuation. The outer constellation points of this clipped and filtered signal, which lie within a certain region which does not affect the BER, are left unaltered, hence the constellation is said to be extended. The remaining constellation points are returned to their original position (before the clipping and filtering process). The outer constellation points have a maximum constellation extension limit $(L)$ and this limit for this particular case is $L=1.4$ (as recommended in the DVB-T2 standard). This iterative POCS approach was terminated after 30 iterations, since this proved to be a convergence point. The clipping threshold and filter parameters were determined after an exhaustive search.

Similarly, the POCS approach was used in the TR method. Each sub-carrier in the TR method is limited to ten times the average power of the data carriers (as recommended in the DVB-T2 standard). The TR signal is oversampled by a factor of 4 , with a clipping threshold of $7.8 \mathrm{~dB}$. This iterative POCS approach used for the TR method was terminated after 60 iterations, since this proved to be a convergence point. Furthermore, in all the BER results which follow, a 64-QAM Gray-coded $8 \mathrm{k}$ mode of the DVB-T2 standard was used to transmit OM-OFDM, OFDM, and clipped OFDM data through a 5-tap typical-urban frequency selective fading channel. For an OM-OFDM, OFDM, and clipped OFDM transmission, CSI is extracted from the pilot symbols and used during the equalization process to mitigate the effects of fading. The pilot symbol placement, as well as TR sub-carrier (used in TR), can be found in the DVB-T2 
standard. Similarly, the 5-tap typical-urban area model was obtained from Patzold [30] (which originates from the COST 207 models). Identical throughput and bandwidth occupancies were used to ensure a fair comparison between the various methods. The OM-OFDM method as well as the other methods conform to both throughput and the spectrum mask properties imposed by DVB-T2 standard. Perfect carrier and timing synchronization is assumed. The parameters used for the OM-OFDM transmission are given in Table 5.

\subsection{A BER performance analysis}

OM-OFDM, OFDM, and clipped OFDM data were sent through a 5-tap typical-urban area by using the parameters previously mentioned.

The averaged PAPR of an OFDM transmission when using the 8k mode of the DVB-T2 standard according to simulations is $13 \mathrm{~dB}$. This PAPR value has also independently been verified in the study of [33]. This PAPR is fixed for an OFDM transmission and may only be changed by adopting one or all of the methods discussed in Section 1. When using the same DVB-T2 standard OM-OFDM allows the PAPR of the signal to be varied, while still maintaining identical throughput and bandwidth occupancy as an OFDM transmission. A direct comparison, as shown in Figure 12, between OFDM and OM-OFDM can be made when both methods offer the same PAPR $(13 \mathrm{~dB})$. From this OM-OFDM and OFDM comparison, it is noted that both methods offered similar BER characteristics at a PAPR of $13 \mathrm{~dB}$. The OM-OFDM method, in addition, allows the designer to vary the PAPR until a desired BER is achieved. A further comparison between OM-OFDM and a clipped OFDM transmission shows that the clipped OFDM transmission reaches a BER

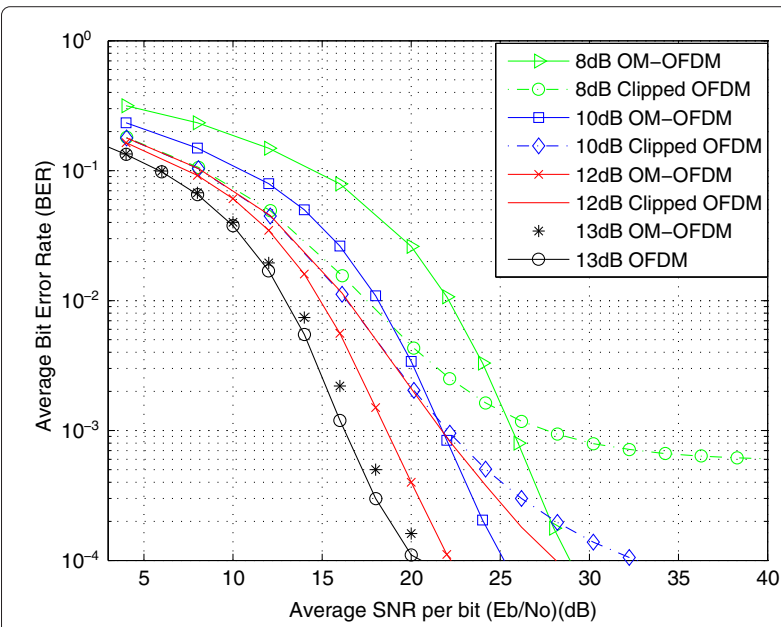

Figure 12 A 64-QAM constellation, BER comparison between an OM-OFDM, OFDM, and a clipped OFDM transmission in a 5-tap typical-urban area. plateau (CRs $\leq 9 \mathrm{~dB})$, whereas OM-OFDM does not result in this BER plateau effect for this case.

When a signal is clipped, information about the signal is permanently removed. Methods like DAR clipping [5], as previously discussed, have been recommended to be used to reconstruct the clipped method, i.e., restore missing information about the signal. However, this DAR method does not work well under frequency selective fading conditions. This permanent removal of information about the signal during clipping results in the subsequent BER plateau effect. A combination of the removal of information about the signal and the channel effects, results in the subsequent clipping BER characteristics. OM-OFDM on the other hand does not remove information about the transmission, hence no BER plateau effect. However, the resultant BER characteristics are primarily dependent on the channel, hence, the various difference between an OM-OFDM and clipped transmission.

Also similarly to the previous case, for the ACE and TR methods the resultant fixed average PAPR is $12 \mathrm{~dB}$ and $12.7 \mathrm{~dB}$, respectively. The BER performance of the ACE and TR methods are not presented, since it resembles that of an OFDM transmission.

\subsection{A decision metric performance analysis}

In order to facilitate a direct comparison between OMOFDM, OFDM, ACE, TR, and a clipped OFDM transmission, the decision metric discussed in Section 5 was employed. The results from such an implementation, depicted in Figure 13, were obtained by using this metric and employing a standard OTS FPD2000AS [37] RF power amplifier, a $10^{\text {th }}$ degree polynomial was used to describe the PAE for this particular amplifier. In Figure 14 the PAE as well as the input output characteristics of such an amplifier are depicted.

When using this standard OTS power amplifier, as depicted in Figure 15, the optimum operating point for an OM-OFDM transmission is at a PAPR of $10 \mathrm{~dB}$ (where a minimum decision metric occurs) and for the ACE and clipped OFDM transmission the optimum operating points are $12 \mathrm{~dB}$. Similarly, the TR transmission has an optimum operating point at $12.7 \mathrm{~dB}$. At these optimum operating points OM-OFDM offers a $1.2 \mathrm{~dB}(23.6 \%)$, $2 \mathrm{~dB}(36.8 \%), 2.2 \mathrm{~dB}(39.8 \%)$, and $4.1 \mathrm{~dB}(60.8 \%)$ net power performance gain (at a BER of $10^{-4}$ ) when compared to an ACE, TR, OFDM, and clipped OFDM transmission, respectively. Hence, the OM-OFDM method has offered a performance improvement when compared to an ACE and TR methods, without the need for an iterative (30-60 iterations) process. Furthermore, the decision metric suggests that the OM-OFDM method's PAPR value may be lowered to $8 \mathrm{~dB}$ (thus a $5 \mathrm{~dB}$ PAPR reduction when compared to the original OFDM transmission), while still maintaining a performance improvement when 


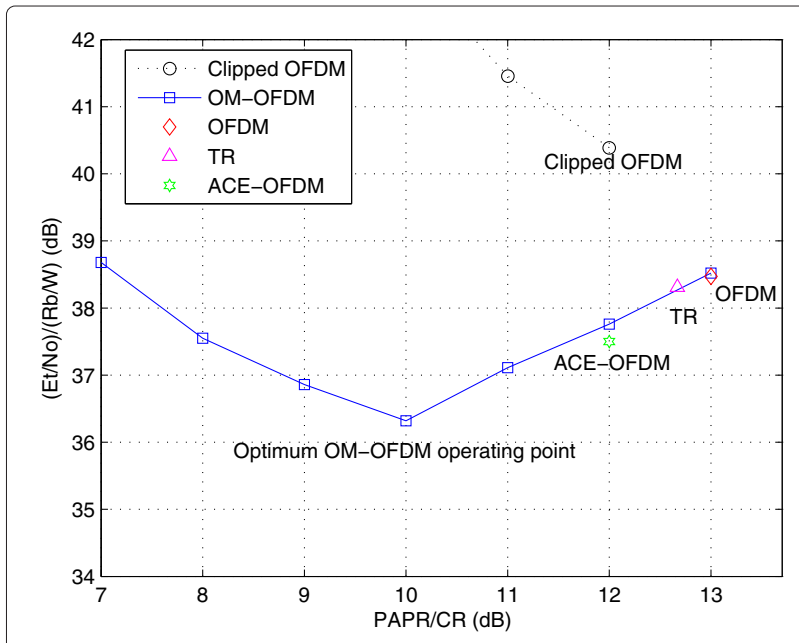

Figure 13 System performance for a 64-QAM constellation at a BER of $10^{-4}$ for an FPD2000AS RF power amplifier.

compared to an ACE, TR, OFDM, and clipped OFDM transmission.

This decision metric result might appear to be misleading, since in Figure 12 at a BER of $10^{-4}$, a $2.2 \mathrm{~dB}$ net gain is not expected, as proposed by the decision metric. This $2.2 \mathrm{~dB}$ net power performance gain is attributed to the fact that the PAE curve of a typical amplifier is exponentially shaped, depicted in Figure 14, instead of linear. Hence, there is an exponential relationship between PAPR $(\mathrm{dB})$ and PAE, instead of a linear relationship. Thus as the PAPR decreases, this leads to an exponential increase in efficiency; this relationship is valid within a certain PAPR range. It is this association which leads to the $2.2 \mathrm{~dB}$ net power performance gain.

In order to further validate the results another standard OTS AN10858 [33] RF power amplifier manufactured by

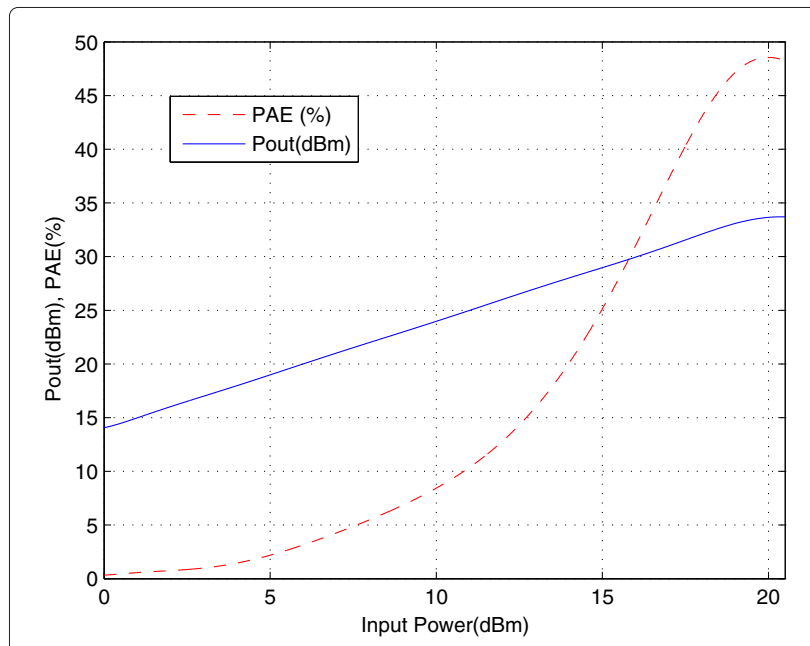

Figure 14 Power compression curves of an FPD2000AS amplifier.

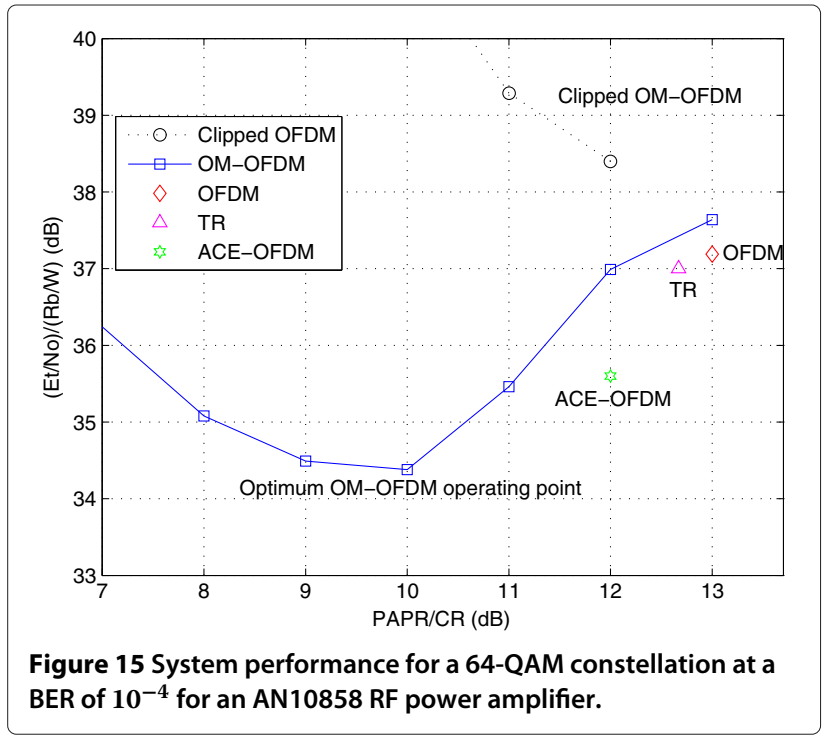

a different supplier was used. A second degree polynomial was used to describe the PAE of this particular amplifier.

Similar to the previous case for this AN10858 standard OTS power amplifier, depicted in Figure 13, at the optimum operating points OM-OFDM offers a $1.2 \mathrm{~dB}(24.6 \%)$, $2.65 \mathrm{~dB}$ (45.3\%), $2.81 \mathrm{~dB}$ (47.60\%), and $4 \mathrm{~dB}(60.34 \%)$ net power performance gain (at a BER of $10^{-4}$ ) when compared to an ACE, TR, OFDM, and clipped OFDM transmission, respectively. Hence, the OM-OFDM method has once again offered a performance improvement when compared to an ACE and TR method. Furthermore, the decision metric suggests that the OM-OFDM method's PAPR value may be lowered to $8 \mathrm{~dB}$, while still maintaining a performance improvement. In Table 6 , the various decision metric results obtained when using an FPD2000AS and an AN10858 RF power amplifier are summarized.

From these comparisons, it is noted that OM-OFDM offers between $4 \mathrm{~dB}-1.2 \mathrm{~dB}(60.34 \%-24.6 \%)$ and $4.1 \mathrm{~dB}-$ $1.2 \mathrm{~dB}(60.8 \%-23.6 \%)$ performance improvement for an AN10858 and an FPD2000AS RF power amplifier, respectively, when compared to an ACE, TR, OFDM, and a clipped OFDM transmission.

Table 6 A decision metric performance improvement obtained when using OM-OFDM at a BER of $10^{-4}$

\begin{tabular}{lll}
\hline & \multicolumn{2}{c}{ Amplifiers } \\
\cline { 2 - 3 } Method & AN10858 & FPD2000AS \\
\hline OFDM & $2.8 \mathrm{~dB}(47.6 \%)$ & $2.2 \mathrm{~dB}(39.8 \%)$ \\
Clipping & $4.0 \mathrm{~dB}(60.4 \%)$ & $4.1 \mathrm{~dB}(60.8 \%)$ \\
ACE & $1.2 \mathrm{~dB}(23.6 \%)$ & $1.2 \mathrm{~dB}(23.6 \%)$ \\
TR & $2.7 \mathrm{~dB}(45.3 \%)$ & $2.0 \mathrm{~dB}(36.8 \%)$ \\
\hline
\end{tabular}




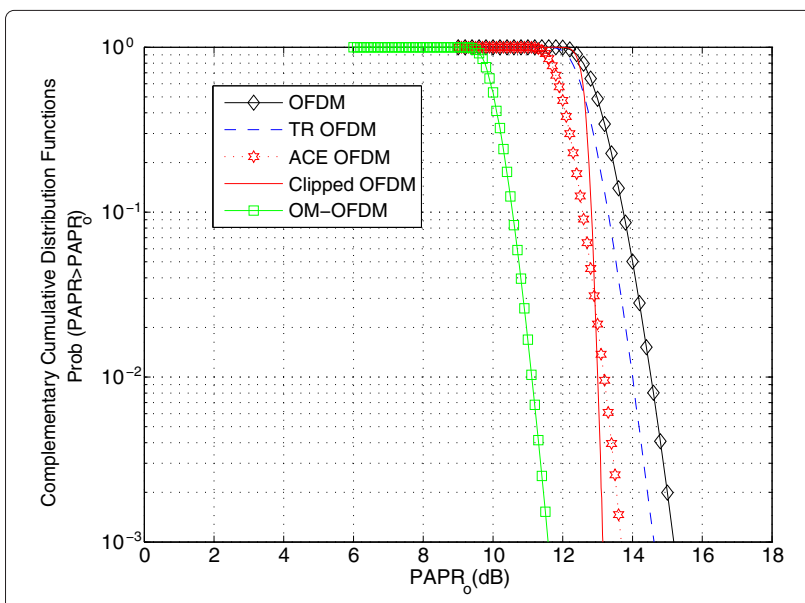

Figure 16 Complementary cumulative distribution functions of an OM-OFDM, OFDM, ACE and TR transmission for a 64-QAM constellation.

\subsection{A CCDF performance analysis}

Based on the optimum operating points obtained from the decision metric in the previous section, the CCDF, depicted in Figure 16, was used to compare the PAPR characteristics of an OM-OFDM, OFDM, clipped OFDM, $\mathrm{TR}$, and ACE transmission.

At these optimum operating points, OM-OFDM is shown to offer a $2 \mathrm{~dB}, 2.27 \mathrm{~dB}, 2.75 \mathrm{~dB}$, and $3.19 \mathrm{~dB}$ PAPR reduction (at a CCDF of $10^{-1}$ ) when compared to an ACE, clipped OFDM, TR, and OFDM transmission, respectively. From these comparisons, it is noted that OM-OFDM offers a significant PAPR reduction when compared to the various methods.

\section{Conclusion}

The authors have proposed a method called offset modulation to control the PAPR of an OFDM transmission. The theoretical bandwidth occupancy of the proposed offset modulation signal was derived. Using these bandwidth occupancy results, a closed-form theoretical BER expression for an offset modulation transmission is derived. This mathematically derived BER expression has been shown to agree with the simulated results, thus validating the derivation. A BER comparison between OM-OFDM and OFDM at a PAPR value of $13 \mathrm{~dB}$ shows that both methods offer similar BER characteristics for frequency selective fading channel conditions. The authors have further introduced a newly applied power performance decision metric, which can be used throughout the PAPR field to compare various methods. This decision metric is used to investigate whether the proposed OM-OFDM transmission has an optimum solution and whether a net gain exists for such a solution. When using this decision metric, OM-OFDM is shown to offer between $4 \mathrm{~dB}-1.2 \mathrm{~dB}(60.34 \%-24.6 \%)$ and $4.1 \mathrm{~dB}-$ $1.2 \mathrm{~dB}(60.8 \%-23.6 \%)$ net power performance gain (at a BER of $10^{-4}$ ) for an AN10858 and an FPD2000AS RF power amplifier, respectively, when compared to a clipped OFDM, OFDM, TR, and ACE transmission, in a frequency selective fading channel. Finally, by using a CCDF, the OM-OFDM method is shown to offer between 3.2 and $2 \mathrm{~dB}$ PAPR reduction (at a CCDF of $10^{-1}$ ) when compared to an OFDM, TR, clipped, and ACE OFDM transmission.

The proposed offset method is shown to offer a performance improvement when compared to both simple (clipping), as well as more well-established (ACE and TR) PAPR reduction methods. These performance gains combined with the fact that OM-OFDM requires lowimplementation complexity and does not lead to a severe BER degradation as the number of carriers increase. It also does not require any additional bandwidth expansion or the transmission of any side information to reconstruct the original message signal. These aspects make it a good alternative approach to current methods already in the field.

\section{Competing interests}

This work is currently being patented by the University of Pretoria. Both authors are inventors on the patent, with the University of Pretoria holding the patent rights. This paper is based on work, for which the author has been awarded a PhD at the University of Pretoria. The necessary permissions have been obtained to publish such material.

Received: 6 February 2012 Accepted: 22 December 2012

Published: 30 January 2013

\section{References}

1. SH Han, JH Lee, An overview of peak-to-average power ratio reduction techniques for multicarrier transmission. IEEE Wirel. Commun. Mag. 12(2), 56-65 (2005)

2. T Jiang, Y Wu, An overview: peak-to-average power ratio reduction techniques for OFDM signals. IEEE Trans. Broadcast. 54(2), 257-268 (2008)

3. K Dhuness, An Offset Modulation method used to control the PAPR of an OFDM transmission. PhD thesis, University of Pretoria, Pretoria, 2012

4. H Ochiai, $\mathrm{H}$ Imai, Performance of the deliberate clipping with adaptive symbol selection for strictly band-limited OFDM systems. IEEE J. Sel. Areas Commun. 18(11), 2270-2277 (2000)

5. D Kim, GL Stuber, Clipping noise mitigation for OFDM by decision-aided reconstruction. IEEE Commun. Lett. 3, 4-6 (1999)

6. AE Jones, TA Wilkinson, SK Barton, Block coding scheme for reduction of peak to mean envelope power ratio of multicarrier transmission schemes. IET Electron. Lett. 30(8), 2098-2099 (1994)

7. JA Davis, J Jedwab, Peak-to-mean power control in OFDM, Golay complementary sequences, and Reed-Muller codes. IEEE Trans. Inf Theory. 45(7), 2397-2417 (1999)

8. RW Bauml, RFH Fischer, JB Huber, Reducing the peak-to-average power ratio of multicarrier modulation by selected mapping. IET Electron. Lett. 32(22), 2056-2057 (1996)

9. CL Wang, Y Ouyang, Low-complexity selected mapping schemes for peak-to-average power ratio reduction in OFDM systems. IEEE Trans. Signal Process. 53(12), 4652-4660 (2005)

10. M Breiling, SH Muller-Weinfurtner, JB Huber, SLM peak-power reduction without explicit side information. IEEE Commun. Lett. 5(6), 239-241 (2001)

11. X Wang, TT Tjhung, CS Ng, Reduction of peak-to-average power ratio of OFDM system using a companding technique. IEEE Trans. Broadcast. 45(3), 303-307 (1999) 
12. X Huang, J Lu, J Zheng, KB Letaief, J Gu, Companding transform for reduction in peak-to-average power ratio of OFDM signals. IEEE Trans. Wirel. Commun. 3(6), 2030-2039 (2004)

13. T Jiang, G Zhu, Nonlinear companding transform for reducing peak-to-average power ratio of OFDM signals. IEEE Trans. Broadcast. 50(3), 239-241 (2004)

14. BS Krongold, DL Jones, PAR reduction in OFDM via active constellation extension. IEEE Trans. Broadcast. 49(3), 258-268 (2003)

15. BS Krongold, DL Jones, An active-set approach for OFDM PAR reduction via tone reservation. IEEE Trans. Signal Process. 52(2), 495-508 (2004)

16. SC Thompson, JG Proakis, JR Zeidler, in Proceedings of the IEEE Military Communications Conference, vol. 1. Constant envelope binary OFDM phase modulation, (Boston, USA, 2003), pp. 621-626

17. SC Thompson, AU Ahmed, JG Proakis, JR Zeidler, in Proceedings of the IEEE Military Communications Conference, vol. 2. Constant envelope OFDM phase modulation: spectral containment, signal space properties and performance, (Monterey, USA, 2004), pp. 1129-1135

18. SC Thompson, JG Proakis, JR Zeidler, vol. 1. Noncoherent reception of constant envelope OFDM in flat fading channels, (Berlin, Germany, 2005), pp. 517-521

19. Y Tsai, G Zhang, JL Pan, in Proceedings of the IEEE Military Communications Conference, vol. 4. Orthogonal frequency division multiplexing with phase modulation and constant envelope design, (Atlantic City, USA, 2005), pp. 2658-2664

20. M Kiviranta, A Mammela, D Cabric, DA Sobel, RW Brodersen, in Proceedings of the IEEE Military Communications Conference, vol. 2. Constant envelope multicarrier modulation: performance evaluation in awgn and fading channels, (Atlantic City, USA, 2005), pp. 807-813

21. SC Thompson, Constant envelope phase modulation. PhD thesis, University of California, San Diego, 2005

22. SC Thompson, JG Proakis, JR Zeidler, M Geile, in Proceedings of the IEEE Military Communications Conference, vol. 1. Constant envelope OFDM in multipath rayleigh fading channels, (Washington, USA, 2006), pp. 1-7

23. AU Ahmed, SC Thompson, JR Zeidler, in Proceedings of the IEEE Military Communications Conference, vol. 1. Constant envelope OFDM with channel coding, (Washington, USA, 2006), pp. 1-7

24. SC Thompson, AU Ahmed, JG Proakis, JR Zeidler, MJ Geile, Constant envelope OFDM. IEEE Trans. Commun. 56(8), 1300-1312 (2008)

25. K Dhuness, BTJ Maharaj, Comparative performance of OM-OFDM in broadband systems. IET Electronic Letters. 48(2), 127-129 (2012)

26. RE Zeimer, WH Tranter, Principles of Communications: Systems, Modulation, and Noise, 3rd edn. (Houghton Mifflin Co International Inc, Washington DC, 1990)

27. K Dhuness, BTJ Maharaj, in Proceedings of the IEEE 72nd Vehicular Technology Conference, vol. 1. An offset modulation scheme to control the PAPR of an OFDM transmission -invited paper, (Ottawa, Canada, 2010), pp. 1-5

28. K Dhuness, BTJ Maharaj, in Proceedings of the IEEE Africon, vol. 1. A cognitive radio application of OM-OFDM for implementation in DVB-T2 —outstanding paper award, (Livingston, Zambia, 2011), pp. 1-6

29. ETSI EN 302 755: Digital Video Broadcasting (DVB); Frame structure channel coding and modulation for a second generation digital terrestrial television broadcasting system (DVB-T2). European Telecommunication Standard Doc. 302, 2009

30. M Patzold, Mobile Fading Channels. (John Wiley and Sons, New York, 2002)

31. JG Proakis, M Salehi, Communication Systems Engineering, 2nd edn. (Prentice-Hall, Upper Saddle River, NJ, 2002)

32. JG Proakis, Digital Communication, 4th edn. (McGraw-Hill, New York, 2002)

33. N Semiconductors, AN10858.pdf, (2010). [http://www.nxp.com/ documents/application_note/]

34. C Liang, J Jong, WE Stark, JR East, Nonlinear amplifier effects in communication systems. IEEE Trans. Microwave Theory Tech. 47(8), 257-268 (1999)
35. A Gatherer, M Polley, in Proceedings of the 32nd IEEE Asilomar Conference on Signals, Systems and Computers, vol. 1. Controlling clipping probability in DMT transmission, (Pacific Grove, CA, USA, 1998), pp. 578-584

36. DL Jones, in Peak power reduction in OFDM and DMT via active channel modification, vol. 2, (Monterey, CA, USA, 1999), pp. 1076-1079

37. RM Devices, FPD2000ASDS.pdf, (2009). [http://www.rfmd.com/CS/ Documents/]

doi:10.1186/1687-1499-2013-19

Cite this article as: Dhuness and Maharaj: Analysis of an offset modulation transmission. EURASIP Journal on Wireless Communications and Networking 2013 2013:19.

\section{Submit your manuscript to a SpringerOpen ${ }^{\circ}$ journal and benefit from:}

- Convenient online submission

- Rigorous peer review

- Immediate publication on acceptance

- Open access: articles freely available online

- High visibility within the field

- Retaining the copyright to your article

Submit your next manuscript at $\boldsymbol{\wedge}$ springeropen.com 